\title{
AN EXAMPLE IN SUMMABILITY
}

\author{
BY W. K. HAYMAN AND ALBERT WILANSKY
}

Communicated by Edwin Hewitt, July 18, 1961

A matrix $A$ is called conservative if $A x$ is convergent (its limit is called $\lim _{A} x$ ) whenever $x$ is a convergent sequence, regular $\operatorname{if~} \lim _{A} x$ $=\lim x$ for such $x$, coregular if conservative and $\chi(A) \equiv \lim _{n \rightarrow \infty} \sum_{k=1}^{\infty} a_{n k}$ $-\sum_{k=1}^{\infty} a_{k} \neq 0$ (here $a_{k}=\lim _{n \rightarrow \infty} a_{n k}$ ), and conull if $\chi(A)=0$. The terms coregular and conull were introduced in [2].

A regular matrix is coregular, as is any matrix equipotent with a regular one. However, there exist coregular matrices not equipotent with any regular matrix. The example, due to Zeller, is given in [3]. We present here an example of a quite different nature.

(An open problem in the field is that of characterizing $F K$ spaces which have a right to be called coregular. That $\{1\}$ be separated from the linear closure of $\left\{\delta^{n}\right\}$ is necessary but not sufficient.)

Restricting ourselves, for convenience, to triangles $\left(a_{n n} \neq 0, a_{n k}=0\right.$ for $k>n)$, let $c_{A}=\{x: A x$ is convergent $\}$. Then $c_{A}$ is isomorphic with $c$, the space of convergent sequences, under $A: c_{A} \rightarrow c$. Thus $c_{A}$ becomes a Banach space. If $c_{A}=c_{B}=F$ say, the norms on $F$ due to $A, B$ are equivalent since $A=D B$ with $c_{D}=c$ and $\|x\|_{A}=\|A x\| \leqq\|D\|\|B x\|$ $=\|D\|\|x\|_{B}$.

If the functional lim is continuous on $c \subset c_{A}$ we extend it by the Hahn-Banach theorem to be defined on all of $c_{A}$. By a construction of Mazur [1, Theorem 2, p. 45], we obtain a matrix $B$ with $\lim _{B}$ $=\lim$ on $c$, and $c_{B}=c_{A}$. (See [2] for proof that lim satisfies Mazur's condition.)

Clearly $B$ is regular.

Conversely if such regular $B$ exists it follows that $\lim$ is continuous since $\lim =\lim _{B}$.

Thus, for our example, it is sufficient to construct a coregular matrix $A$ such that lim is not continuous on $c_{A}$.

Let $Y$ be the matrix such that $Y x=\left\{x_{n-1}+x_{n}\right\}$. Then (1/2) $Y$ is a regular triangle. Let $B$ be the matrix whose $n$th row is $\left\{t_{1}, t_{2}, \cdots\right.$, $\left.t_{n-1}, 0,0, \cdots\right\}$ where $\left\{t_{n}\right\}$ is a suitably chosen sequence with $\sum\left|t_{n}\right|<\infty$. Then $B$ is in the radical of the Banach algebra $\Delta$ of conservative triangular matrices. (See [4].) Note that $Y$ has no inverse in this algebra. Finally, let $A=B+Y$. Then $A$ is coregular. The norm associated with $c_{A}$ is

$$
\|x\|=\sup _{n}\left|\sum_{k=1}^{n-1} t_{k} x_{k}+x_{n-1}+x_{n}\right| .
$$


We shall chouse $t_{n}=(-1)^{n} / n^{2}$. Now to construct $x$ with $|\lim x|$ large and $\|x\|$ not large we shall, given any integer $m$, supply $x$ with $\lim x=-1 / 2 \log m,\|x\|<M$ where $M$ is an absolute constant which could easily be determined.

Namely, let

$$
\begin{aligned}
x_{n} & =n(-1)^{n}-1 / 2 \log n \quad \text { for } 1 \leqq n \leqq m, \\
& =(2 m-n)(-1)^{n}-1 / 2 \log m \quad \text { for } m<n \leqq 2 m, \\
& =-1 / 2 \log m \quad \text { for } n>m .
\end{aligned}
$$

If the matrix $Y$ had been chosen so that $Y x=\left\{s x_{n-1}+t x_{n}\right\}$ then for $t>s \geqq 0$ we have that $Y^{-1} \in \Delta$ hence $A^{-1} \in \Delta$ since $B$ is in the radical. Thus $c_{A}=c, \lim$ is continuous and $\|x\|<1$ implies $|\lim x|$ is less than some constant, depending on $s, t$ and $\left\{t_{n}\right\}$. This result also follows from Lemma 4.2 of [4]. There should be situations between these extremes in which $c_{A} \neq c$, yet a regular matrix coincident with $A$ exists. This may very well occur if all $t_{n}>0$, and $s=t=1$, but we are unable to say at present.

Added in proof. Research Problem 3 (Bull. Amer. Math. Soc. vol. 67 (1961) p. 355) by Albert Wilansky, which inspired this article, has also been solved by Lawrence Shepp.

\section{REFERENCES}

1. S. Mazur, Eine Anwendung der Theorie der Operationen bei der Untersuchung der Toeplitzchen Limitierungsverfahren, Studia Math. vol. 2 (1930) pp. 40-50.

2. A. Wilansky, An application of Banach linear functionals to summability, Trans. Amer. Math. Soc. vol. 67 (1949) pp. 59-68.

3. - Summability: the inset, replaceable matrices, the basis in summability space, Duke Math. J. vol. 19 (1952) pp. 647-660.

4. A. Wilansky and K. Zeller, Banach algebra and summability, Illinois J. Math. vol. 2 (1958) pp. 378-385.

IMPERIAL COLLEGE, LONDON, AND

LEHIGH UNIVERSITY 